\title{
RHIC orbit response analysis with LOCO Run 12
}

\section{T. Summers, J. Kewisch}

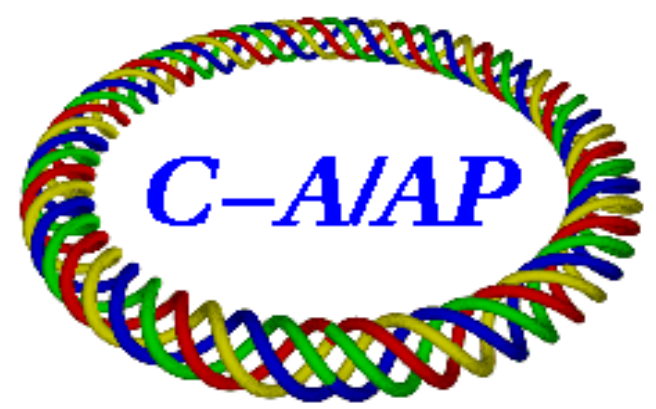

\section{Collider-Accelerator Department Brookhaven National Laboratory Upton, NY 11973}

Notice: This document has been authorized by employees of Brookhaven Science Associates, LLC under Contract No. DE-AC02-98CH10886 with the U.S. Department of Energy. The United States Government retains a nonexclusive, paid-up, irrevocable, world-wide license to publish or reproduce the published form of this document, or allow others to do so, for United States Government purposes. 


\section{RHIC Orbit Response Analysis with LOCO Run 12}

T. Summers, J. Kewisch. BNL-CAD. March 21, 2013.

\section{Introduction}

Orbit response matrix analysis (ORM) is a well-established technique used to find and correct linear optics errors in accelerators. The measurement consists of recording the change in the closed orbit around the ring in response to successively changing dipole corrector magnets by a known amount. The analysis is performed by adjusting parameters in the accelerator model until the difference between the modeled response matrix and the measurement is minimized. Typical parameters to adjust are quadrupole and skew quadrupole strength, dipole corrector magnet gains and rolls and BPM gains and rolls. The adjusted model should be a close approximation to the real machine as seen by the BPMS. The calculated changes in gain/strength can be applied to the real machine to return the linear optics to the design.

In Rhic Run 12 three sets of orbit response matrices were measured and analyzed using the Matlab-based LOCO program and lattices derived from RampDesigner's sxf models. Analyses were performed with emphasis on fitting the triplet and trim quadrupoles. While none of the solutions were tested in the machine, the measurements have been useful for finding deficiencies in the model and for testing the various options in LOCO. It is challenging to find a solution without unrealistically large oscillating fits between neighboring quadrupoles; a degeneracy likely due to the small phase advance between the quads.

This effort is a continuation of the work started by T. Satogata in 2006. In AP Note \#267 [1] he describes the ORM technique and the result of simulations on the pp35 lattice. He concluded that with reasonably low BPM noise ( 5 um), LOCO should be able to resolve random $0.1 \%$ gradient errors on all the quadrupoles as well as $5 \%$ gain errors on the BPMS and correctors.

\section{Analysis Tools}

\subsection{Accelerator Toolbox and LOCO}

The analysis program LOCO [2] is installed with the Accelerator Toolbox [3] package in Matlab. These programs are freely available online [4]. At CAD, they are installed in $\sim m c r / M a t l a b / A T$, with orm data and scripts in $\sim$ mcr/Matlab/AT/machine/RHIC/orm.

\subsection{Modifications to Matlab Model Lattice}

Matlab model lattice files are made from the sxf files using a Perl script RhicSxfToAT.pl. A template of this script is located in the /orm directory. In each of the specific lattice folders within /orm for which a measurement was taken is a copy of this RhicSxfToAT script, tailored to that lattice. For example, /orm/CuAu12injection/RhicSxfToATCUAu12inj.pl gives the skew quad strength file, lists the faulty BPMs at the time of the measurement and sets the feed-down offset for each ring.

\subsubsection{Feed-down in Dipoles}

We have modified the sbend definition to include feed-down effects. Previously, the sbend definition did not include any quadrupole or sextupole terms (the DX dipoles are an exception for they include thin focusing kicks at the end facing away from the IP to account for the large design orbit offset). Each sbend in the sxf file gives 
quadrupole and sextupole component strengths for the full magnet as well as the sextupole component for the entry and exits.

In Matlab we have added a sextquad to the entry and exit of each sbend. It has the sextupole strength $S$ from the $s x f$ file as well as the quadrupole feed-down (-2* $S^{*}$ offset) and a dipole component $\left(S^{*}\right.$ offset $\left.{ }^{2}\right)$. A new sbend definition, sbends $x$, was created to include the sextupole term $S$, the quadrupole term $K$ (as $K-2 * S^{*}$ offset) as well as an extra dipole term $\left(K^{*}\right.$ offset $+S^{*}$ offset $\left.^{2}\right)$.

The offset is empirically determined so the tunes in the model are correct (the Rhic online model uses $-1.5 \mathrm{~mm}$ ). For the yellow ring at injection the offset is $-2.34 \mathrm{~mm}$ for both the CuAu12-v1 lattice and the pp12b-v2 lattice (without the snake). With the CuAu12-v1 lattice in the blue ring at injection it is $-2.18 \mathrm{~mm}$, for the blue ring at store with the same lattice it is $-8.2 \mathrm{~mm}$; the large magnitude of this offset at store needs to be investigated.

\subsubsection{Snake magnet transfer matrices}

For polarized proton lattices, the snakes have been added as a $6 \times 6$ transfer matrix. The matrices were calculated with MAD by cutting the snake magnets into $2401 \mathrm{~cm}$ slices and rotating each slice by the appropriate tilt angle. The focusing is about the same for both snakes.

$\begin{array}{rrrrrrr}\text { snkMat } & =0.962046 & 10.817463 & 0.001315 & -0.000001 & 0.000000 & -0.000314 \\ -0.006884 & 0.962046 & 0.000000 & -0.001315 & 0.000000 & -0.000072 \\ 0.001315 & 0.000001 & 0.962049 & 10.818477 & 0.000000 & 0.122567 \\ 0.000000 & -0.001315 & -0.006883 & 0.962049 & 0.000000 & -0.000430 \\ 0.000072 & 0.000314 & -0.000430 & 0.122567 & 1.000000 & 0.004032 \\ 0.000000 & 0.000000 & 0.000000 & 0.000000 & 0.000000 & 1.000000\end{array}$

\subsubsection{Skew quad strengths}

As the skew quadrupole strengths are not given in the sxf file, a program was made to get the values from the $a p p \_s t o r e / R u n D a t a / . . . / R a m p / S t e p S t o n e s /$ archive. These strengths can now be loaded into the matlab lattice. Instructions can be found in the RhicSxfToAT.pl templates.

\section{Run 12 Experiments}

\subsection{Measurement and Analysis Procedure}

The procedure for taking measuring an orbit response matrix is detailed in lorm/documents/RHICOrmProcedure.docx.

Essentially, the measurement is automated once the machine is tuned to a nominal state. This means the orbit, tune and coupling feedback has been run, the chromaticity set to near the design values. If both rings are to be measured simultaneously the rings should be anti-cogged or filled with a pattern that has longitudinal separation built in. All the measurements taken have had either 6 or 12 bunches per ring, with nominal bunch intensity. Typically, the IR separation bumps are removed to provide more room. 


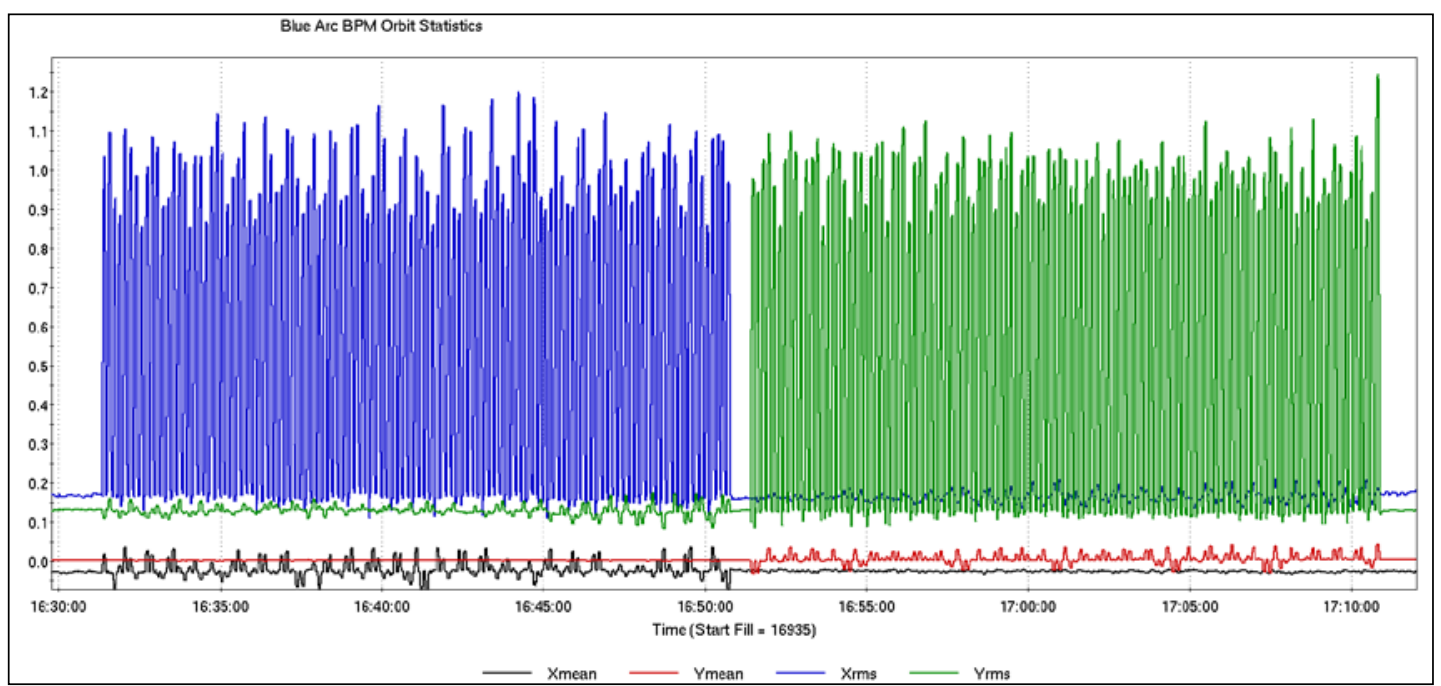

Figure 1: Sample ORM measurement as seen by arc BPMs. Each spike is the rms orbit due to changing one dipole corrector by an amount calculated to give a $1 \mathrm{~mm}$ response. The horizontal response is done first, followed by the vertical. The duration of the measurement (performed at injection energy for the CuAu12v1 lattice) is about 40 minutes.

The measurement script measureORM.py uses the online model to calculate the strength change for every dipole corrector in order to achieve an RMS orbit in the arcs of (default) $1 \mathrm{~mm}$, an example of a complete measurement is seen is Figure 1. Each corrector is changed in order of s-coordinate starting at 6 o'clock and after a delay of 3-4 s for the magnet to settle the orbit timestamp is recorded in a log file. Another script, ORManalyze.py uses the log file to assemble the orbit response matrix into a text file. If the measured dispersion is to be used in the analysis it needs to be saved from the RHICchromaticity application to a single column text file.

The analysis is performed with the LOCO program. Input files are created to gather all the necessary files - lattice, measurement, dispersion, bpms/correctors, which quadrupole gradients and other parameters (skew quads, magnet tilt, number of singular values, etc.) to use in the fitting. The fit parameters are varied until the difference between the modeled response matrix and the measured response matrix is minimized. For each iteration, the fitted parameters are updated to the local model and can be saved to the matlab workspace for plotting. The quality of the minimization is determined by the number of singular values used, the number of outliers removed, the $\chi^{2} /(n$ degrees of freedom), the magnitude of the difference between the modeled and measured response matrices.

\subsection{Scheduled APEX Time}

On three occasions during RHIC Run 12 an ORM measurement was taken. In the following cases there was no attempt to apply a calculated correction back to the machine due to the large changes to the quadrupoles that LOCO calculated. We expect the changes to the quadrupole strengths to be on the order of a few $10^{-4} \mathrm{~m}^{-1}$.

\subsubsection{March 21, pp12b-v2::injection, yellow ring}

Scheduled 2 hours, 12 bunches with $1.25 \mathrm{e} 11 \mathrm{pp} /$ bunch, tunes corrected to $\mathrm{x}=28.695$ and $y=29.685$. This was the first time taking a measurement with new measureorm.py script. Two small bugs in the script were found and corrected - factor of $\sqrt{ } 2$ resulted in RMS orbit difference in arcs of $1.6 \mathrm{~mm}$ instead of requested $1.0 \mathrm{~mm}$ and the log file recorded the total corrector strength instead of the corrector change. 'MeasuredOrm032112.txt' and other relevant data, including dispersion measurements saved to /orm/pp12binjection.

For this first measurement, neither sextuple feed-down dipoles nor the snakes were included in the model lattice. For the analysis, gradients of the insertion focusing/defocusing quads $1,2,3,7,8,9$ as well as trim quads 4,5,6 were varied. The figure of merit $\chi^{2} / n d f$ was reduced in 2 iterations from 1115 to 88 using all 225 singular values. 


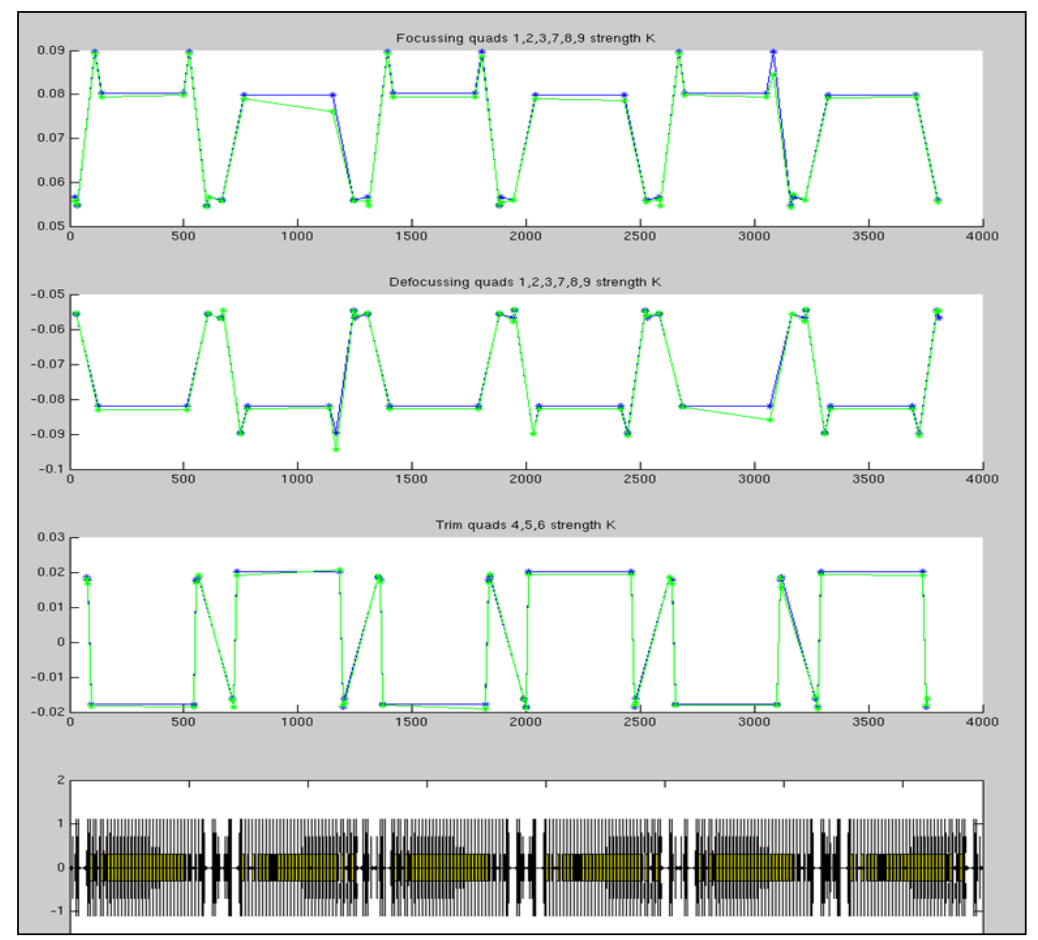

Figure 2: Insertion quadrupole strengths for initial model (blue) and after fitting (green). $\mathrm{x}$-axis is s-position around the yellow ring, starting at 6 o'clock.

Immediately evident in the fitted quadrupole strengths in Figure 2 is the effect of the snakes - LOCO tries to change the nearby quads by up to $6 \%$. This indicated the need to find a way to include the snakes in the model. This was done, as described in section 2.2.2, but this did not improve the results of the analysis as much as was hoped there remained large, alternating quadrupole values, especially in the area of the snakes.

\subsubsection{May 16, CuAu12-v1s::store, blue ring}

While work was taking place to establish Au beam in the injectors, $\mathrm{Cu}$ beam was available at store in the blue ring. The machine had 12 bunches with $1 \mathrm{e} 9 \mathrm{Cu} /$ bunch, tunes were corrected and the separation bumps removed. We requested $1 \mathrm{~mm}$ rms in arcs with the response at IRs $6 \& 8$ up to $6 \mathrm{~mm}$ due to large store beta functions. 'MeasuredOrm051612.txt' and other relevant data saved to /orm/Cu12store.

As with the previous analysis, LOCO finds changes to quadrupole gradients (Figure 3 ) to greatly reduce the difference between the modeled and measured response matrices (Figure 4). Again, the size of the gradients is large - few $10^{-3}$ for the triplets. Also of concern is how in many of the IRs the 2 triplet quads of the same polarity have large corrections of opposite sign. This is likely due to the very small phase advance between these quads. There are many options and methods available in LOCO to try combat these degenerate solutions, and many were tried with this data set. Unfortunately, we have not been able to arrive at a promising solution.

In [5] "LOCO with Constraints and Improved Fitting Technique" the authors describe the common degeneracy problem and describe two methods to try combat it. First, they recommend using the Levenberg-Marquadt method over the Gauss-Newton method when the initial difference between model and machine is too large. Secondly, they describe how to add cost factors to the fitting parameters. Both of these methods have been tried but so far have not yielded more reasonable solutions. 


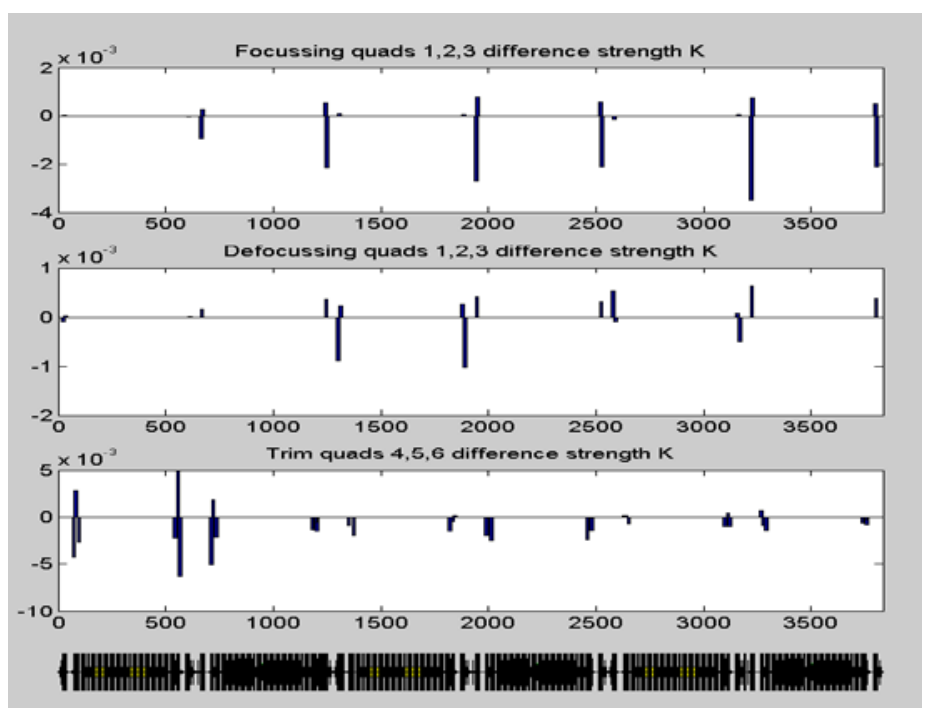

Figure 3: Changes to quadrupole strengths found by LOCO for the blue ring at store. Top plot are the focusing triplet quads, middle plot are the defocusing triplet quads and the bottom plot are the trim quads $(4,5,6)$.
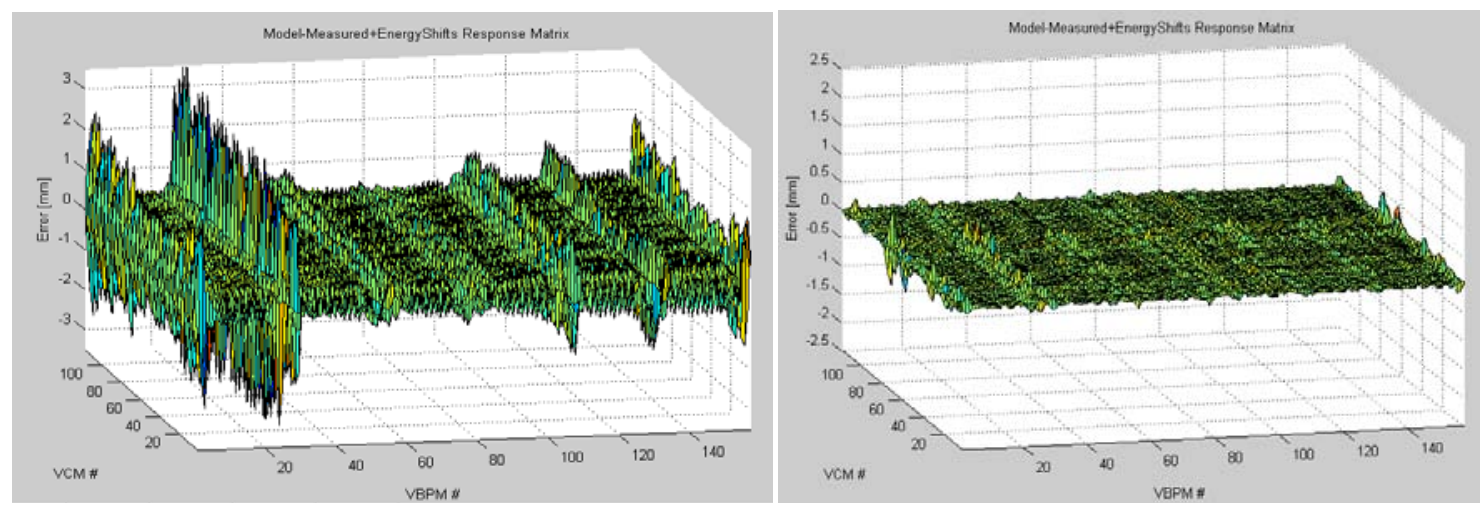

Figure 4: Model minus measured resposne matrices for the vertical plane before (left) and after (right) applying the quadrupole changes in Figure 3. Horizontal plane is similar.

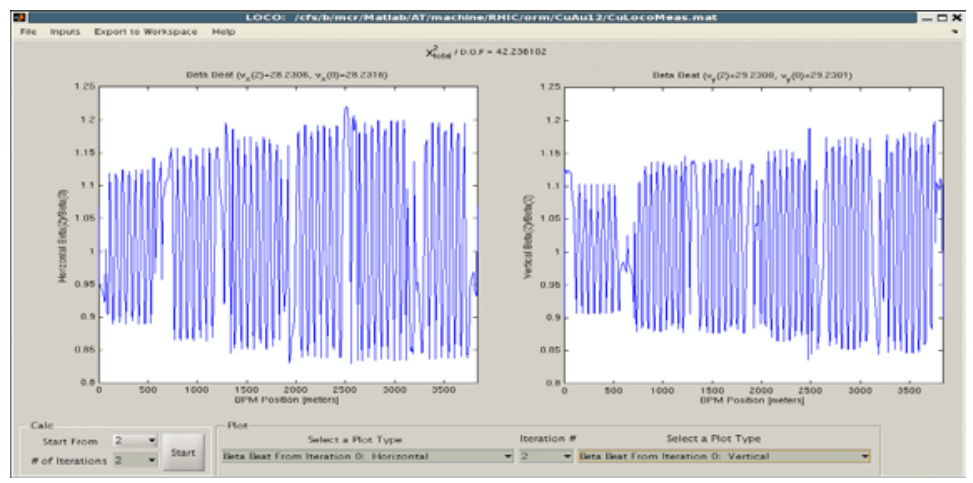

Figure 5: Calculated beta beat due to quadrupole settings shown in Figure 3.

\subsubsection{May 30, CuAu12-v1::injection, both rings}

Because the results of the first 2 measurements had unexpectedly large quadrupole strengths it was decided to set up a measurement to try and find quadrupoles with known errors. Two measurements were taken during this time: one on the nominal machine and one after changing four quadrupoles in each ring. The difference between the 2 LOCO solutions should only be the quadrupole errors in Table 1. 


Quad changes (Matlab K, RampEditor KL):
\begin{tabular}{|c|c|c|c||}
\hline & Matlab & RampEditor & Length(m) \\
\hline \hline bi9-tq6 & $+\mathbf{0 . 0 0 1}$ & $+\mathbf{0 . 0 0 0 7 5}$ & $\mathbf{0 . 7 5}$ \\
\hline \hline bi9-tq4 & $-\mathbf{0 . 0 0 4 4 2}$ & $-\mathbf{0 . 0 0 3 3 2}$ & $\mathbf{0 . 7 5}$ \\
\hline bo3-tq5 & $+\mathbf{0 . 0 0 1}$ & $+\mathbf{0 . 0 0 0 7 5}$ & $\mathbf{0 . 7 5}$ \\
\hline \hline bi4-tq5 & $+\mathbf{0 . 0 0 1 1 6 3}$ & $+\mathbf{0 . 0 0 0 8 7 2}$ & $\mathbf{0 . 7 5}$ \\
\hline \hline yi7-qf3 & $+\mathbf{0 . 0 0 0 2 5}$ & $+\mathbf{0 . 0 0 0 5 2 5}$ & $\mathbf{2 . 1 0}$ \\
\hline \hline yi7-qf1 & $-\mathbf{0 . 0 0 1 9 8 5}$ & $\mathbf{- 0 . 0 0 2 8 6}$ & $\mathbf{1 . 4 4}$ \\
\hline \hline yil1-qd2 & $+\mathbf{0 . 0 0 0 2 5}$ & $+\mathbf{0 . 0 0 0 8 4 7 5}$ & $\mathbf{3 . 3 9}$ \\
\hline \hline yo12-qf2 & $+\mathbf{0 . 0 0 0 2 1 1}$ & $+\mathbf{0 . 0 0 0 8 7 2}$ & $\mathbf{3 . 3 9}$ \\
\hline
\end{tabular}

Table 1: Size of changes to 4 quadrupoles in each ring for 2 nd measurement.

For the Yellow ring ( $\mathrm{Au}$ ), fit Q/D 1,2,3 and TQ 4,5,6 as well as the bpm and corrector gains and coupling. The quadrupole fits are plotted in Figure 6 for both measurements, and the difference between the 2 measurements can be seen. While LOCO nearly finds the 4 quads that were changed, there are many other quadrupoles with differences between the 2 fits of the same magnitude.

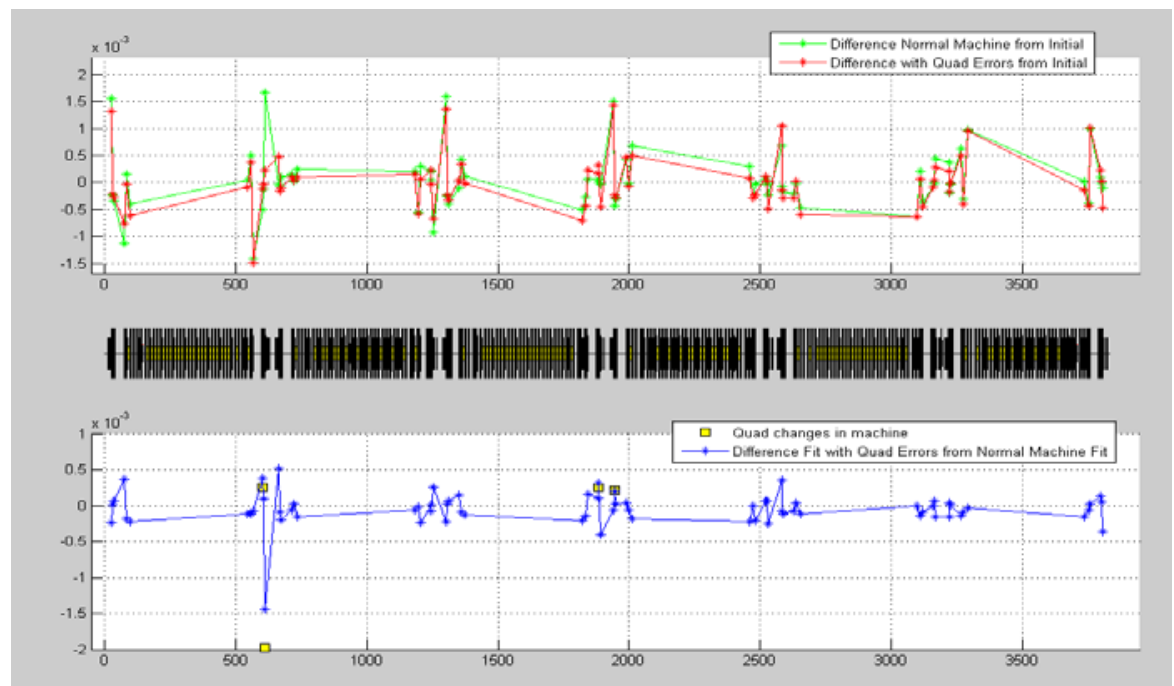

Figure 6: Quadrupole strength fits for the yellow ring, top plot: in green are the fitted strengths from the nominal machine, in red are the fitted strengths from the machine with the 4 quad errors. The bottom plot shows the difference between the two - the yellow squares are the 4 quad changes that were input.

An example of the fitted corrector magnet gain and coupling is plotted in Figure 7 and the fitted BPM gain and coupling is plotted in Figure 8. In all cases, the blue trace is the fit for the measurement on the nominal machine and the green trace is the fit for the measurement on the machine with the quadrupole errors. There is great agreement between the 2 measurements, except for the $5 \%$ scaling difference between the BPMs and correctors in the 2 nd measurement. 

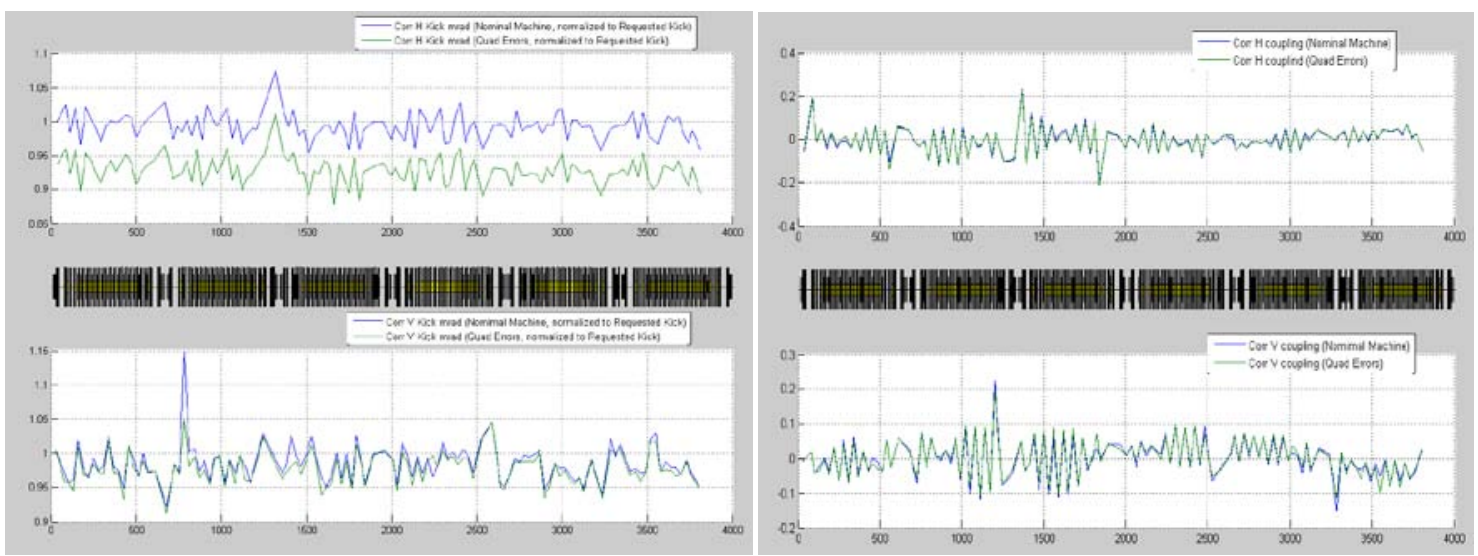

Figure 7: Left: corrector gain fits and right: corrector coupling; in each the top is horizontal and the bottom is vertical, the blue is the fit from the measurement on the nominal machine and the green in the fit from the measurement on the machine with 4 quad errors.
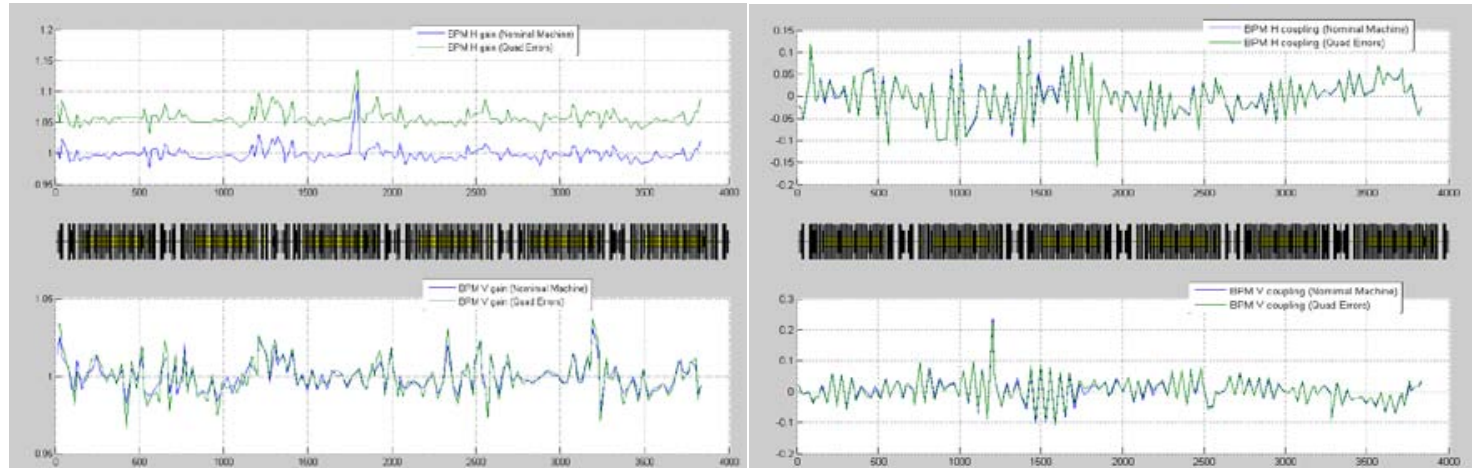

Figure 8: Left: BPM gain fits and right: BPM coupling; in each the top is horizontal and the bottom is vertical, the blue is the fit from the measurement on the nominal machine and the green in the fit from the measurement on the machine with 4 quad errors.

The measurements in the blue ring $(\mathrm{Cu})$ were fitted in the same manner as the measurements in the yellow ring, fit Q/D 1,2,3 and TQ 4,5,6. In the blue ring the 4 quad errors were located in the trim magnets (in the yellow ring the 4 errors were in the triplets). The resulting quadrupole strength difference between the two measurements is shown in Figure 9. LOCO appears to finds these errors reasonably well except for changing all three time quads in 9 o'clock instead of just the 2 that were changed.

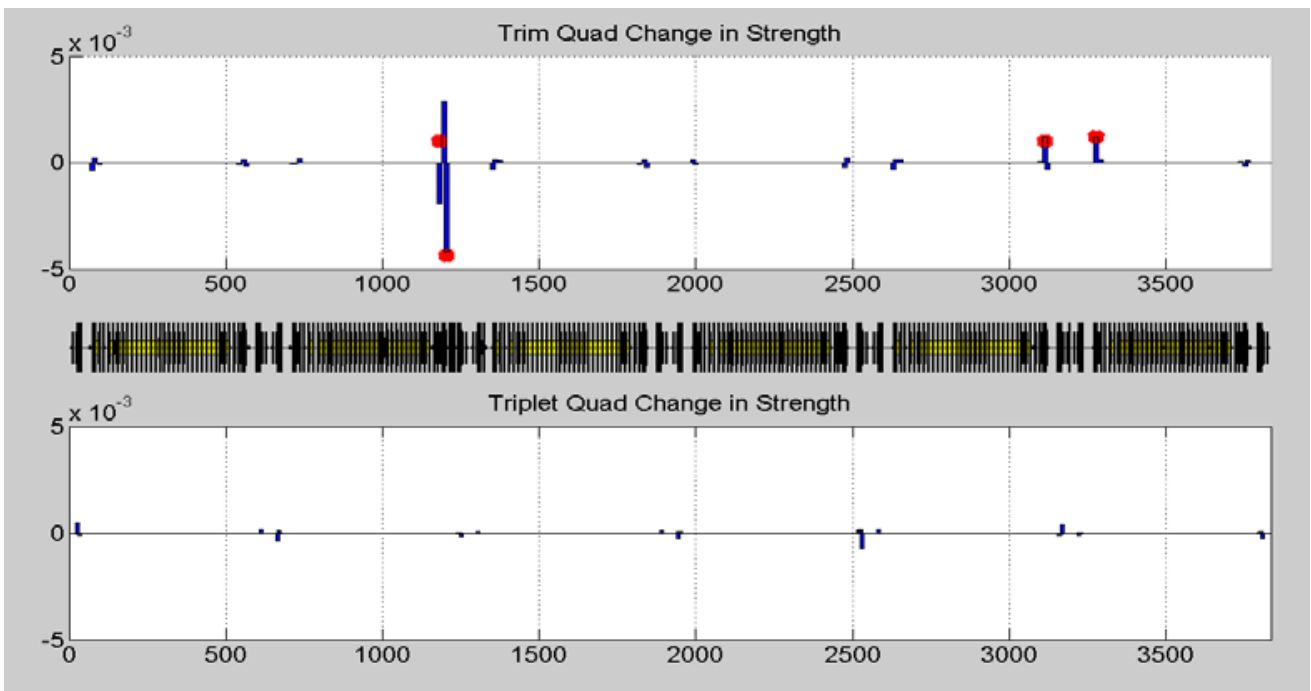

Figure 9: Difference between the fitted quadrupole strength from the measurement on the nominal machine and the measurement on the machine with the 4 trim quad errors. Red dots are the intentional errors. 
To test whether the solution found was equivalent to the machine with the changes, the beta beat in each case was calculated. In Figure 10 it is seen that the 2 cases are, for the large part, not equivalent.

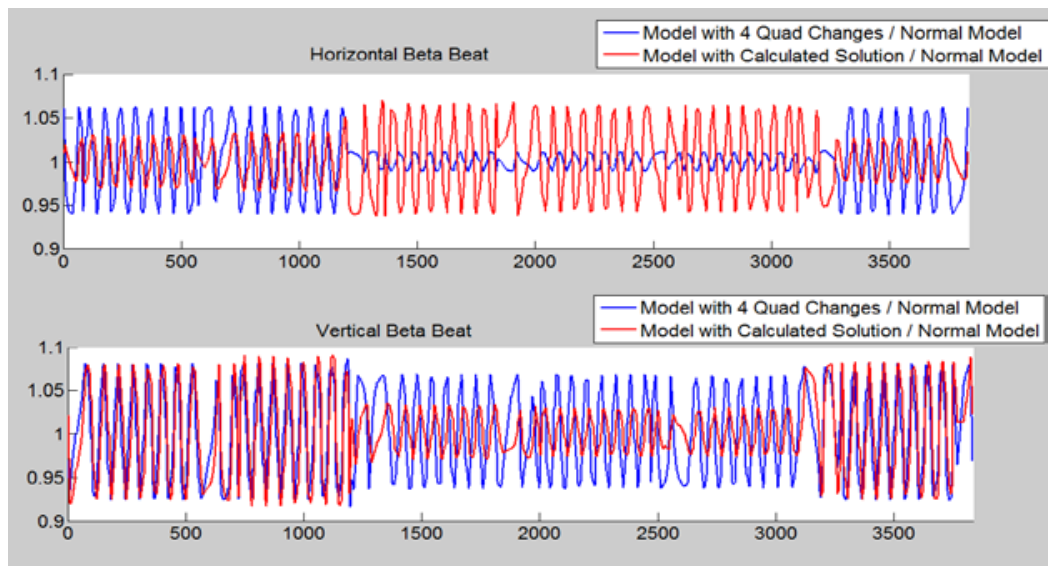

Figure 10: Calculated beta beat for (blue) the model with the 4 quad changes and for (red) the model with the calculated solution.

\section{Conclusions}

In Run 12 we had the opportunity to measure 4 response matrices, two of which were on the same machine with the only difference being intentional changes to four quadrupoles in each ring. From the 1st measurement, taken with the polarized proton injection lattice, we learned that the model lattice must include the snake magnets and we have found a way to add them. We found that LOCO seems to find a solution to minimize the difference between the measured and model response matrices but the size of the changes to the quadrupoles it calculates are unrealistically large. The 2 nd measurement was the only measurement taken at store and again, although the program finds solutions that minimize the difference between the model and the measurement the changes in quadrupole strength are too large. The last set of data we measured, comparing the machine with and without the errors showed that although we can almost find the intentional errors, the overall size of the correction is very large and that the resulting solution does not necessarily produce the same effect on beta beats.

There are many options available in the LOCO program. Many of them have been tried while attempting to find reasonable solutions but we have not yet found a combination that works. More investigation is required.

\section{References}

[1] T. Satogata and J. Niedziela, "Simulations of RHIC Orbit Response Analysis using LOCO", C-AD AP note 267, Feb 2007.

[2] G. Portmann, J. Safranek, A. Terebilo, "LOCO-MATLAB Manual”, http://wwwssrl.slac.stanford.edu/ safranek/loco/

[3] A. Terebilo, "Accelerator Toolbox for MATLAB", SLAC-PUB-8732, May 2001.

[4] http://als.lbl.gov/als physics/portmann/ 
[5] X. Huang, J. Safranek, G. Portmann, "LOCO with Constraints and Improved Fitting Technique", ICFA Dynamics Newsletter Vol. 44, pp. 60-69, December 2007. 